\title{
New Prospects for an Old Enzyme: Mammalian Cytochrome $c$ Is Tyrosine-Phosphorylated in $\mathrm{Vivo}^{\dagger}$
}

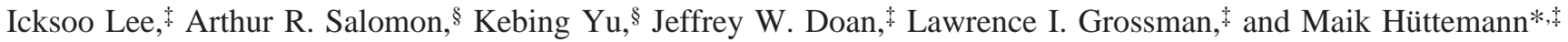 \\ Center for Molecular Medicine and Genetics, Wayne State University School of Medicine, Detroit, Michigan 48201, and \\ Department of Molecular Biology, Cell Biology and Biochemistry, Brown University, Providence, Rhode Island 02912
}

Received March 24, 2006; Revised Manuscript Received May 31, 2006

\begin{abstract}
Mammalian cytochrome $c$ (Cyt $c$ ) has two primary functions: transfer of electrons from the $b c_{1}$ complex to cytochrome $c$ oxidase (COX) as part of the mitochondrial electron transport chain (ETC), and participation in type II apoptosis. Several studies have indicated that components of the ETC can be phosphorylated, and we have recently shown that the Cyt $c$ electron acceptor COX is phosphorylated on Tyr-304 of subunit I in liver upon activation of the cAMP-dependent pathway, leading to strong enzyme inhibition. However, covalent modification of Cyt $c$ through phosphorylation has not yet been reported. We have isolated Cyt $c$ from cow heart under conditions that preserve the physiological in vivo phosphorylation status. Western analysis with an anti-phosphotyrosine antibody indicated tyrosine phosphorylation. The site of phosphorylation was definitively assigned by immobilized metal affinity chromatography/nano-liquid chromatography/electrospray ionization mass spectrometry (IMAC/nano-LC/ ESI-MS) to Tyr-97, one of the four tyrosine residues present in Cyt $c$. The phosphorylated tyrosine is part of a motif that contains five residues identical to the tyrosine phosphorylation site in COX subunit I. Spectral analysis revealed that the characteristic $695 \mathrm{~nm}$ absorption band is shifted to $687 \mathrm{~nm}$ and reversed after treatment with alkaline phosphatase. This band results from the Met-80-heme iron bond, and its shift might indicate changes in the catalytic heme crevice. In vivo phosphorylated Cyt $c$ shows enhanced sigmoidal kinetics with COX, and half-maximal turnover is observed at a Cyt $c$ substrate concentration of $5.5 \mu \mathrm{M}$ compared to $2.5 \mu \mathrm{M}$ for alkaline phosphatase-treated Cyt $c$. Possible consequences of Tyr-97 phosphorylation with respect to cardiolipin binding and of location of Tyr-97 in close proximity to Lys-7, a crucial residue for interaction with Apaf-1 during apoptosis, are discussed.
\end{abstract}

Cytochrome $c(\mathrm{Cyt} c)^{1}$ was first discovered by MacMunn more than a century ago (1) and later rediscovered by Keilin in 1925 (2). Since that time, more than 12000 papers have been published centrally involving Cyt $c$, including the first crystal structures from horse and tuna at $2.8 \AA$ in 1971 (3). Consisting of 104 amino acids in human, cow, and mouse, Cyt $c$ is a redox-active molecule owing to the presence of a heme group covalently linked to Cys-14 and -17 . The heme iron is coordinated by His-18 and Met- 80 .

Cyt $c$ is a multifunctional protein. As part of the mitochondrial electron transport chain (ETC), Cyt $c$ is an isopotential electron carrier accepting one-electron packages from the $b c_{1}$ complex and transferring them to cytochrome $c$ oxidase (COX). In mice, this function becomes essential

$\doteqdot$ The financial support of the Center for Molecular Medicine and Genetics, Wayne State University, Detroit, MI, is gratefully acknowledged.

* To whom correspondence should be addressed at Center for Molecular Medicine and Genetics, Wayne State University School of Medicine, 3127 Scott Hall, 540 E. Canfield, Detroit, MI 48201. Fax: (313) 577-5218. Phone: (313) 577-9150. E-mail: mhuttema@ med.wayne.edu.

Wayne State University School of Medicine.

$\S$ Brown University.

${ }^{1}$ Abbreviations: COX, cytochrome $c$ oxidase; Cyt $c$, cytochrome $c$; ETC, electron transport chain; IMAC/nano-LC/ESI-MS, chromatography/nano-liquid chromatography/electrospray ionization mass spectrometry; SAP, shrimp alkaline phosphatase. after midgestation, as has been shown with Cyt $c$ knockout mice, which die around that developmental stage (4). Switching occurs at midgestation from energy production that relies only $5 \%$ on aerobic metabolism before gestation day 9 to $95 \%$ after gestation day 11, based on studies with cultured rat embryos (5).

A second function of Cyt $c$ is its participation in type II apoptosis, which is triggered by changes of mitochondrial integrity and involves release of Cyt $c$ into the cytosol, activating procaspase- 9 to caspase- 9 via a complex with Apaf-1 (apoptosome) (6). Although mechanistically not fully understood, the release of mitochondrial proteins, in particular Cyt $c$, is considered a very important proapoptotic signal (7-9). As part of this apoptotic process, it has recently been shown that Cyt $c$ exerts a further enzymatic function as a cardiolipin oxygenase: after induction of apoptosis $\mathrm{Cyt}$ $c$, which is attached to negatively charged phospholipids (in particular cardiolipin) of the inner mitochondrial membrane, chemically oxidizes cardiolipin, allowing its detachment and release from the mitochondria (10). A somewhat converse function as a scavenger of reactive oxygen species has been proposed under physiological (nonapoptotic) conditions (11).

Cyt $c$ has additional features, including an ATP binding site that has been mapped to the conventional "left side" of the molecule (12). At physiological concentrations of $5 \mathrm{mM}$ ATP, its binding to Cyt $c$ leads to changes in the binding of 
Cyt $c$ to COX, the inhibition of the reaction between Cyt $c$ and $\mathrm{COX}$, and the elimination of the low $K_{\mathrm{m}}$ phase of the otherwise biphasic kinetics with COX (13).

Despite decades of intensive investigation, one important aspect of Cyt $c$ has been missed thus far: its modification and potential regulation via phosphorylation mediated through cellular signaling pathways. Recent attention has been drawn to signaling pathways targeting the mitochondria, and a few studies have begun to reveal that some components of the mitochondrial oxidative phosphorylation machinery can be phosphorylated in vivo. We have recently shown that liver COX can be phosphorylated on Tyr-304 of catalytic subunit I and that this phosphorylation is mediated through the cAMP-dependent pathway, leading to strong enzyme inhibition (14). Others have shown that COX subunit II is targeted for phosphorylation by Src kinase in osteoclasts, leading to enzyme activation (15). In addition, a recent study has revealed that cow heart NADH dehydrogenase is phosphorylated in vivo, on Ser-59 of subunit NDUFA10 (16). The delta subunit of ATP synthase can also be phosphorylated (17), and there is indirect evidence that this phosphorylation occurs on Tyr-75 (18).

Because of the central role of Cyt $c$ in respiration and apoptosis, we asked whether the molecule can be phosphorylated in vivo. Here, we show for the first time that cow heart Cyt $c$ isolated under conditions that preserve the physiological phosphorylation status is tyrosine-phosphorylated, and that such phosphorylation affects the Cyt $c$ spectrum and the electron-transfer kinetics with COX. We discuss potential implications of this novel feature including control of respiration and the role of Cyt $c$ in apoptosis, mediated through cell signaling pathways.

\section{MATERIALS AND METHODS}

Isolation of Heart Mitochondria and Cyt c. Chemicals were purchased from Sigma, unless otherwise stated. Mitochondria were isolated at $4{ }^{\circ} \mathrm{C}$ or on ice from cow heart tissue as described (14) with small modifications. Briefly, $300 \mathrm{~g}$ of tissue was ground and further homogenized with a commercial blender in a 5 -fold volume of buffer A $(250 \mathrm{mM}$ sucrose, $20 \mathrm{mM}$ Tris ( $\mathrm{pH} 7.4$ ), and $2 \mathrm{mM}$ EDTA). To maintain the physiological phosphorylation status, this buffer was supplemented with $1 \mathrm{mM}$ vanadate, $10 \mathrm{mM} \mathrm{KF}$, and 2 $\mathrm{mM}$ EGTA. After centrifugation $(650 \mathrm{~g}, 10 \mathrm{~min})$, the supernatant was collected through a piece of cheesecloth. The pellet was homogenized and centrifuged one more time to increase the yield of mitochondria. Combined supernatants were centrifuged (16 300g, $20 \mathrm{~min}$ ). The mitochondria were resuspended in $200 \mathrm{~mL}$ of buffer A using a Teflon homogenizer (150 rpm, 4 strokes), and buffer A was added to a volume of $2.5 \mathrm{~L}$ and centrifuged at low speed ( $370 \mathrm{~g}$ for 5 $\min )$ to remove contaminants. The supernatant was centrifuged $(16300 \mathrm{~g}, 20 \mathrm{~min})$ to collect mitochondria, which were washed one more time by resuspending and centrifuging (16 300 $\mathrm{g}, 20 \mathrm{~min})$. Cyt $c$ was released by adding $10 \mathrm{vol}$ of $120 \mathrm{mM} \mathrm{KH} \mathrm{PO}_{4}, \mathrm{pH} 7.4$, to the washed mitochondria, followed by sonication (micro tip, $4 \times 30 \mathrm{~s}$ pulses). The suspension was centrifuged for $60 \mathrm{~min}$ at $195000 \mathrm{~g}$, and the Cyt $c$-containing supernatant was purified via two-step ion exchange chromatography modified from ref 19 . The solution was adjusted to $\mathrm{pH} 7.5$, diluted with water until a conduc- tance of $4.8 \mathrm{mS} / \mathrm{cm}$ was reached, and applied to a DEAE anion exchange column (GE Healthcare) equilibrated with $\mathrm{KH}_{2} \mathrm{PO}_{4}, \mathrm{pH} 7.5$, of similar conductance (about $20 \mathrm{mM}$ ). More than $80 \%$ of all proteins bind to the column; the flowthrough, containing the Cyt $c$ (19), was diluted to a conductance of $2 \mathrm{mS} / \mathrm{cm}$, adjusted to $\mathrm{pH} 6.5$, and applied to a CM sepharose cation exchange column (Whatman) equilibrated with $\mathrm{KH}_{2} \mathrm{PO}_{4}, \mathrm{pH} 6.5,2 \mathrm{mS} / \mathrm{cm}$ conductance. Under those conditions, Cyt $c$ binds to the column, which was then washed with 4 vol of equilibration buffer. A three-step

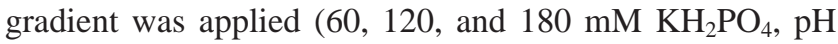
6.5) in which Cyt $c$ eluted in the last fraction. Cyt $c$ was concentrated under vacuum to $1 \mathrm{~mL}$ and further purified and desalted using Sephadex G50 (GE Healthcare) gel filtration

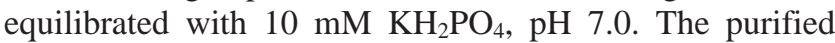
protein was stored at $-80{ }^{\circ} \mathrm{C}$.

Dephosphorylation of Cyt $\mathrm{c}$. Five hundred microliters of a $40 \mu \mathrm{M} / \mathrm{L}$ Cyt $c$ solution was incubated with shrimp alkaline phosphatase (10 units, Roche) overnight at $30^{\circ} \mathrm{C}$. The sample was then desalted using NAP-10 columns (GE Healthcare) equilibrated with $50 \mathrm{mM}$ Tris, $\mathrm{pH}$ 7.5, and Cyt $c$ separated from the phosphatase by applying the solution to a Microcon YM-100 centrifugal device (Millipore). Cyt $c$ was collected in the flow-through.

Western Analysis. SDS-polyacrylamide gel electrophoresis (SDS-PAGE) of Cyt $c$ was carried out using a 4-12\% gradient NuPAGE Bis-Tris gel (Invitrogen). EGF-stimulated A431 cell lysate (Upstate) was included as a positive control and ovalbumin as a negative control. Protein bands were visualized using the Silver Stain Plus kit (Bio-Rad) according to the manufacturer's instructions. For Western analysis, protein transfer time on a nitrocellulose membrane $(0.2 \mu \mathrm{m})$ was $5 \mathrm{~min}$ to prevent transfer through the membrane. Small and highly charged proteins can easily be lost during the multiple washing steps. Thus, to prevent Cyt $c$ dissociation, we incubated the membrane in a $1 \%$ glutaraldehyde solution for $10 \mathrm{~min}$, and subsequently applied three UV-cross-linking pulses (0.12 J each, UV Stratalinker 1800, Stratagene). This combination was found to be optimal with respect to Cyt $c$ retention on the membrane. Because amines, such as Tris, compete with the chemically formed cross-links (Schiff base), all solutions subsequently used contained 20 mM HEPES, $\mathrm{pH} 7.5$, instead of an amine-based buffering reagent. Antiphosphoserine and anti-phosphothreonine antibodies were sets of four $(1 \mathrm{C} 8,4 \mathrm{~A} 3,4 \mathrm{~A} 9$, and 16B4) and three (1E11, 4D11, and 14B3) individual monoclonal antibodies (Calbiochem), respectively, whereas a single anti-phosphotyrosine antibody was used (4G10, Upstate). Western analysis was performed with a 1:5000 dilution of anti-phospho antibodies followed by a 1:5000 dilution of anti mouse $\mathrm{IgG}$ alkaline phosphatase-conjugated secondary antibody (Bio-Rad). Signals were detected via the colorimetric method using nitro blue tetrazolium chloride (NBT) and 5-bromo-4-chloro-3indolyl phosphate (BCIP) (Roche).

Spectral Analysis of Cyt $c$. Cyt $c$ was oxidized with 100 $\mathrm{mM} \mathrm{K}_{3} \mathrm{Fe}(\mathrm{CN})_{6}$ and desalted using NAP-10 columns equilibrated with $50 \mathrm{mM}$ Tris, $\mathrm{pH}$ 7.5. Spectra were recorded on a Jasco V-550 double beam spectrophotometer (2 nm bandwidth; $400 \mathrm{~nm} / \mathrm{min}$ scanning speed). Reduced Cyt $c$ was obtained by addition of $100 \mathrm{mM} \mathrm{Na}_{2} \mathrm{~S}_{2} \mathrm{O}_{4}$, and spectra were acquired after desalting the samples as above. 
Isolation of Cytochrome c Oxidase and Enzymatic Activity Measurements. Regulatory competent cytochrome $c$ oxidase (COX) was isolated from cow liver under conditions preserving the phosphorylation status as previously described (14). The enzyme represents the inhibited (via the cAMP pathway) subunit I Y304 phosphorylated COX. To remove cholate and to replace potentially damaged cardiolipin, COX was dialyzed in the presence of $0.1 \mathrm{mM}$ ATP and a 40 -fold molar excess of cardiolipin in $10 \mathrm{mM} \mathrm{K-HEPES} \mathrm{(pH} \mathrm{7.4),}$ $40 \mathrm{mM} \mathrm{KCl}, 1 \%$ Tween 20, 2 mM EGTA, $10 \mathrm{mM} \mathrm{KF}$ (an unspecific serine/threonine phosphatase inhibitor), and $1 \mathrm{mM}$ vanadate (an unspecific tyrosine phosphatase inhibitor). COX activity was analyzed in a closed $200 \mu \mathrm{L}$ chamber containing a micro Clark-type oxygen electrode (Oxygraph system, Hansatech). Measurements were performed in the presence of $2.5 \mathrm{mM}$ ATP, an allosteric inhibitor of COX, after incubation with an ATP regenerating system: COX was incubated at $35{ }^{\circ} \mathrm{C}$ for $30 \mathrm{~min}$ in $10 \mathrm{mM} \mathrm{K}$-HEPES $(\mathrm{pH}$ 7.4), $40 \mathrm{mM} \mathrm{KCl}, 1 \%$ Tween 20, 2 mM EGTA, $10 \mathrm{mM} \mathrm{KF}$, $2.5 \mathrm{mM}$ ATP, $5 \mathrm{mM} \mathrm{MgCl} 2,5 \mathrm{mM}$ phosphoenolpyruvate, and $10 \mathrm{U} / \mathrm{mL}$ pyruvate kinase. Measurements were carried out using $250 \mathrm{nM} \mathrm{COX}$ at $25^{\circ} \mathrm{C}$ after the addition of ascorbic acid $(20 \mathrm{mM})$ and increasing amounts of purified Cyt $c$ from 0 to $16 \mu \mathrm{M}$. Oxygen consumption was recorded on a computer and analyzed with the Oxygraph software. Turnover $(\mathrm{TN})$ is defined as oxygen consumed $[\mu \mathrm{mol}] /(\mathrm{s} \cdot \mathrm{COX}-$ $[\mu \mathrm{mol}])$.

Immobilized Metal Affinity Chromatography/Nano-Liquid Chromatography/Electrospray Ionization Mass Spectrometry (IMAC/Nano-LC/ESI-MS) of Cow Cyt c. A $0.5 \mathrm{mg}$ fraction of cow heart Cyt $c$ was isolated under conditions preserving the physiological phosphorylation status. Cyt $c$ was denatured in $100 \mathrm{mM} \mathrm{NH}_{4} \mathrm{HCO}_{3}$ buffer, $\mathrm{pH} 8.3$, containing $8 \mathrm{M}$ urea, for $5 \mathrm{~min}$ at $96{ }^{\circ} \mathrm{C}$. The mixture was diluted with an equal volume of water, and proteins were digested overnight with $5 \mu \mathrm{g}$ of modified trypsin (Promega, Madison, WI) at $37^{\circ} \mathrm{C}$. The peptides were loaded onto C18 RP peptide macrotrap cartridges (Michrom Bioresources, Auburn, CA) and washed with $1 \mathrm{~mL}$ of $0.1 \%$ acetic acid to remove urea and salts from the digest. Peptides were eluted with $500 \mu \mathrm{L}$ of $70 \%$ acetonitrile $/ 0.1 \%$ acetic acid and dried under vacuum (Thermo Savant, Holbrook, NY). Dried tryptic peptides were incubated with $2 \mathrm{~N}$ d4-methanolic $\mathrm{DCl}$ under nitrogen prepared by the addition of $120 \mu \mathrm{L}$ of acetyl chloride with stirring to $750 \mu \mathrm{L}$ of methanol-D4 (20). This treatment resulted in the efficient methyl ester derivatization of every Asp, Glu, and peptide C-terminus with a $17.0342 \mathrm{Da} \mathrm{OCD}_{3}$. Phosphopeptides were enriched with an automated desalt/ immobilized metal affinity chromatography (IMAC)/nanoliquid chromatography/electrospray ionization mass spectrometry platform as described previously $(21)$. Peptides were eluted with a 30 min $0-70 \%$ solvent B reversed-phase gradient through an analytical column with integrated $5 \mu \mathrm{m}$ electrospray tip into an LTQ mass spectrometer with $30 \mathrm{~nL} /$ min peak parking (solvent $\mathrm{A}, 0.1 \mathrm{M}$ acetic acid in $\mathrm{ddH}_{2} \mathrm{O}$; solvent B, $0.1 \mathrm{M}$ acetic acid in acetonitrile). Peptides were analyzed by data-dependent tandem mass spectrometry (MS/ MS) experiments using collisionally induced dissociation (Xcalibur 1.4 parameters designated 35\% collision energy, $3 \mathrm{Da}$ isolation window, top 5 data dependent, repeat count of one and a dynamic exclusion time of $1.5 \mathrm{~min}$, LT-MS AGC of $3 \times 10^{4}$, and FT-MS/MS AGC of $1 \times 10^{6}$ ).
$\mathbf{A}$

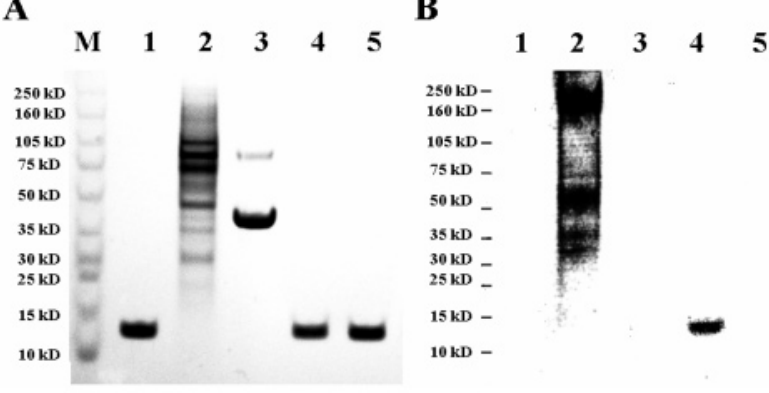

FIGURE 1: Gel and Western analysis of isolated Cyt $c$. (A) Cow heart Cyt $c$ purified under conditions preserving the in vivo phosphorylation status (lane 4) and after treatment with shrimp alkaline phosphatase (SAP, lane 5) was applied to a 4-12\% gradient SDS-PAGE gel, and protein bands were visualized by the silver staining method. Purified Cyt $c$ produces a single band with the expected size (lane 4). Lane M, protein size marker (GE Healthcare); lane 1, Sigma Cyt $c$; lane 2, EGF-stimulated A431 cell lysate (positive control for Western analysis); lane 3, ovalbumin (negative control for Western analysis). (B) For Western analysis, protein samples were applied to SDS-PAGE and subsequently transferred to a nitrocellulose membrane and analyzed using an anti-phosphotyrosine antibody (4G10, Upstate). Purified Cyt $c$ (lane 4) produces a strong signal with the anti-phosphotyrosine antibody, whereas overnight treatment with SAP abolishes the signal (lane 5). Samples in lanes $1-5$ as denoted in panel A. Protein sizes are indicated on the left.

Database Analysis. MS/MS spectra were assigned to peptide sequences from the NCBI nonredundant protein database sliced in Bioworks 3.1 for bovine proteins and searched with the SEQUEST algorithm (22). SEQUEST search parameters designated a static modification of +17.0342 Da on Asp, Glu, and the C-terminus (deuteriomethyl esters) and variable modifications of $+79.9663 \mathrm{Da}$ on Ser, Thr, and Tyr (phosphorylation). Phosphopeptide spectra were manually verified.

Molecular Modeling. Crystallographic data from horse Cyt $c(1 \mathrm{CRC} ; 23)$, which has the same amino acid sequence as cow, was used. The introduction of the phosphate group to Tyr-97 was performed with the program Hyperchem. A geometrically optimized structure for the phosphotyrosine side chain was obtained applying 500 cycles of the "steepest descent" algorithm. For presentation, the modeled Cyt $c$ was processed with the program Swiss PDB viewer (version 3.7). Overlay of the Cyt $c$ and COX phospho-epitopes was performed with the iterative magic fit function and by using crystallographic data from cow heart COX (24).

\section{RESULTS}

Isolation of in Vivo Phosphorylated Cyt c. To maintain the physiological phosphorylation status during mitochondria and subsequent Cyt $c$ isolation, we supplemented all solutions with vanadate, a nonspecific protein tyrosine phosphatase inhibitor, potassium fluoride, a nonspecific inhibitor of protein phosphatases, and EGTA, a calcium chelator, preventing activation of calcium-dependent protein phosphatases. Cyt $c$ of isolated mitochondria was released by breaking the mitochondrial membranes at a high ionic strength to abolish electrostatic interactions of highly positively charged Cyt $c$ with COX, the $b c_{1}$ complex, and the mitochondria-specific phospholipid cardiolipin. Purified Cyt $c$ was then obtained after DEAE and CM ion exchange chromatography followed by Sephadex G50 gel filtration (Figure 1A, lane 4). The 

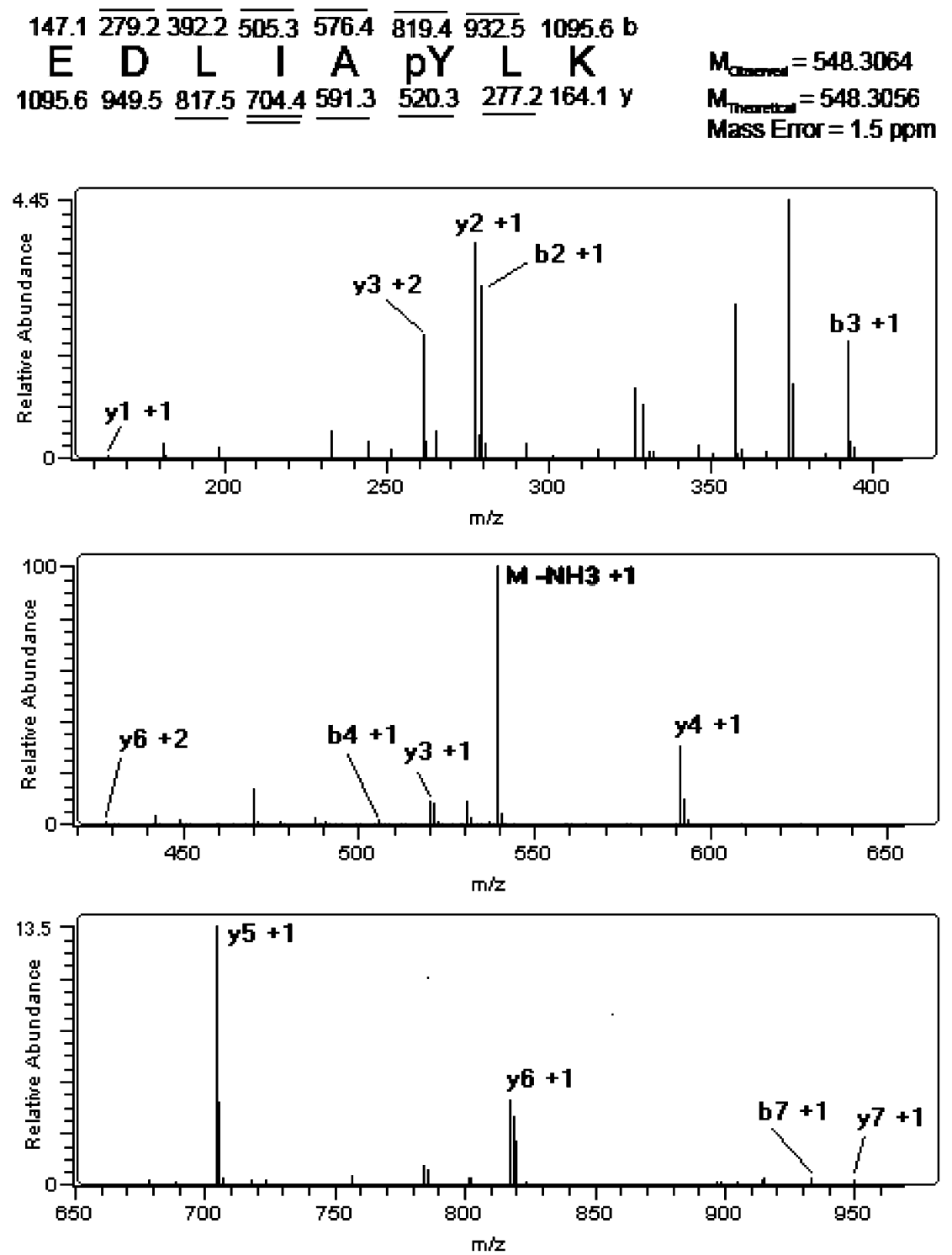

FIGURE 2: Nano-LC/ESI/MS/MS spectrum of EDLIApYLK. Peptides were eluted into the mass spectrometer with an HPLC gradient $(0-50 \%$ acetonitrile, $0.1 \mathrm{M}$ acetic acid in $30 \mathrm{~min})$. The mass spectrometer acquired top 3 data-dependent ESI MS/MS spectra. The phosphorylation site was revealed by fragment ions b2, b3, b4, y5, y6, and y7, and the peptide sequence was assigned by a4, b2, b3, b4, b5, b6, b7, y2, y3, y4, y5, y5 ${ }^{2+}$, and y6.

phosphorylation status was analyzed using a phosphotyrosine-specific antibody, which revealed a strong signal (Figure $1 \mathrm{~B}$, lane 4), indicating that at least one of the four tyrosine residues present in Cyt $c$ was phosphorylated. Incubation of isolated Cyt $c$ with shrimp alkaline phosphatase (SAP) led to dephosphorylation, as was confirmed by Western analysis (Figure 1B, lane 5). Commercially available Cyt $c$ was included as a control (Figure 1B, lane 1) and showed a weak signal only after prolonged detection reaction of the blot (not shown), suggesting that it may contain a minor amount of tyrosine-phosphorylated Cyt $c$ species. The purification method used in producing commercial Cyt $c$ is not available, but due to the weak signal, we assume that phosphatase inhibitors were not included; to our knowledge there is no previously published method for the isolation of Cyt $c$ applying conditions that preserve the phosphorylation state.

Heart Cyt c Is Phosphorylated at Tyr-97. Cyt $c$ isolated under conditions that preserve the in vivo phosphorylation status was fully digested with trypsin with a sequence coverage of $92 \%$, including all four tyrosine residues present in Cyt $c$. Fragment analysis by nano-LC/ESI/MS/MS revealed that the peptide EDLIAYLK contains a phosphate group. The phosphorylation site was unequivocally assigned to Tyr-97 by fragment ions b2, b3, b4, y5, y6, and y7. The sequence of the peptide was definitively assigned by a4, b2, b3, b4, b5, b6, b7, y2, y3, y4, y5, y5 $5^{+}$, and y6 (Figure 2). There was no indication of a second phosphorylation site.

Tyr-97 is located on the conventional "right side" of the molecule (12), and the side chain is essentially solventexposed and thus accessible for a kinase and a phosphatase. Tyr-97 is part of the only true $\alpha$ helical stretch in Cyt $c$, encompassing residues 91-101 (3), and two neighbors of Tyr-97, Leu-94 and Leu-98, are spatially packed against the heme group (25). Tyr-97 is located in the midst of this region filled with hydrophobic amino acids, which has been called the "right channel" (3): it is a closed channel with no solvent access to the heme group, but containing a conduit of hydrophobicity from the heme to the molecular surface, suggesting that it may serve as a binding site for a hydrophobic moiety, for example, a lipid (see Discussion). 


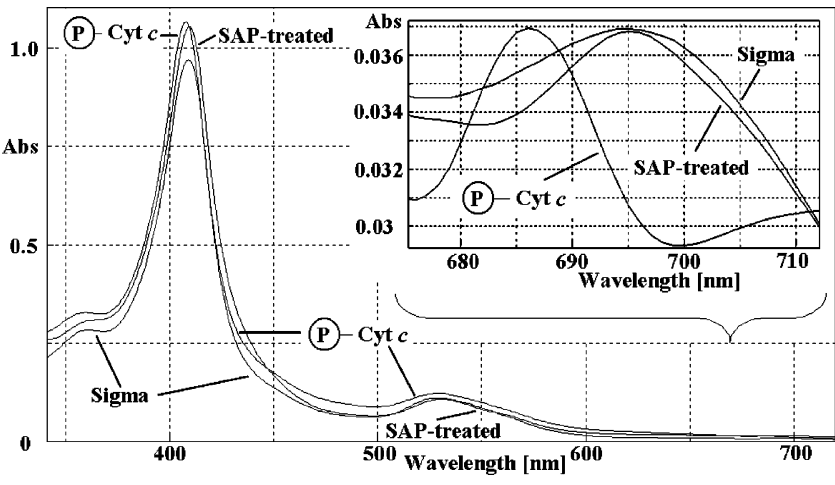

FIGURE 3: Spectral analysis of Cyt $c$ in the oxidized state. UV/vis spectra were recorded on a V550 double beam spectrophotometer (Jasco). Samples were in $50 \mathrm{mM}$ Tris, $\mathrm{pH}$ 7.5. Purified phosphorylated (P-Cyt $c$ ), shrimp alkaline phosphatase-treated (SAP-treated), and commercially available (Sigma) cytochrome $c$ show similar absorption peaks for the $\gamma$-band $(408,409$, and $409 \mathrm{~nm}$, respectively) and the band at 530, 530, and $529 \mathrm{~nm}$, respectively. Interestingly, the characteristic band at $695 \mathrm{~nm}$ is shifted to $687 \mathrm{in}$ the purified phosphorylated Cyt $c$ (inset), which is reversed after treatment with SAP.

Spectral Analysis of Tyr-97-Phosphorylated Cyt c Reveals a Shift of the $695 \mathrm{~nm}$ Band. Cyt $c$ residue Met-80 is a heme ligand, and this bond causes a characteristic $695 \mathrm{~nm}$ absorption band. Only the functional (reducible) state III form shows this band, and it was therefore called a "marker of Cyt $c$ integrity", whereas its absence was called "an indicator of trouble" (26). For example, when Met-80 (and Met-65) is carboxymethylated, it no longer binds to the heme iron, the $695 \mathrm{~nm}$ band is abolished, and Cyt $c$ cannot be reduced by ascorbate nor can it restore respiration in Cyt $c$-depleted rat liver mitochondria or with purified COX (27-31). Interestingly, spectral analysis of purified oxidized heart $\mathrm{Cyt}$ $c$ revealed that the characteristic absorption at $695 \mathrm{~nm}$ is shifted to $687 \mathrm{~nm}$ (Figure 3, inset), which might indicate changes in Cyt $c$ functionality. Removal of the phosphate groups by treatment with shrimp alkaline phosphatase revealed a $695 \mathrm{~nm}$ absorption band very similar to commercially available Cyt $c$, suggesting that phosphorylation causes the spectral change (Figure 3, insert). Reduced purified Cyt $c$ shows only minor spectral differences for the characteristic $\alpha$-, $\beta$-, and $\gamma$-bands $(549,521$, and $413 \mathrm{~nm})$ in comparison to commercially available Cyt $c(550,520$, and $415 \mathrm{~nm}$; not shown).

Phosphorylated Cyt c Produces Pronounced Sigmoidal Kinetics with Cytochrome c Oxidase (COX). To test one possible effect of Cyt $c$ phosphorylation, we analyzed oxygen consumption of purified cow COX with in vivo phosphorylated and SAP-treated Cyt $c$. We found that phosphorylated Cyt $c$ showed pronounced sigmoidal kinetics, whereas SAPdephosphorylated Cyt $c$ produced a hyperbolic response (Figure 4). Although the maximal turnover is $32 \mathrm{~s}^{-1}$ for both in vivo phosphorylated and SAP-treated Cyt $c$, the $K_{\mathrm{m}}$ of COX with each Cyt $c$ species differs by more than 2-fold. It is $5.5 \mu \mathrm{M}$ for phosphorylated and $2.5 \mu \mathrm{M}$ for SAP-treated Cyt $c$.

\section{DISCUSSION}

Our recent finding that $\mathrm{COX}$ is targeted for phosphorylation by cellular signaling pathways in vivo (14) raised the question whether the COX substrate Cyt $c$ could also be

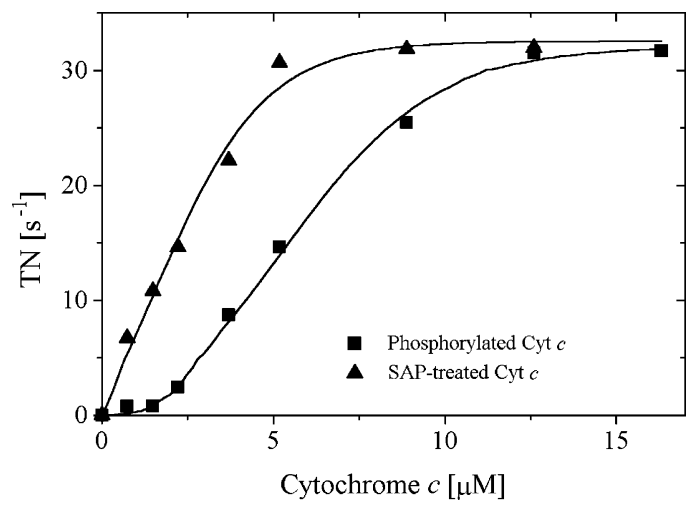

FIGURE 4: Respiration kinetics with in vivo phosphorylated or dephosphorylated cytochrome $c$ and cytochrome $c$ oxidase (COX). Increasing amounts of purified phosphorylated (squares) and shrimp alkaline phosphatase-treated (SAP-treated, triangles) cytochrome $c$ were added to solubilized COX. COX activity was measured with the polarographic method at $25^{\circ} \mathrm{C}$. COX activity (turnover, TN) is defined as consumed $\mathrm{O}_{2}[\mu \mathrm{mol}] /(\mathrm{s} \cdot \mathrm{COX}[\mu \mathrm{mol}])$.

phosphorylated. COX catalytic subunit I residue Tyr-304, which we have shown to be phosphorylated (14), is located in the mitochondrial intermembrane space, as is Cyt $c$. Using a protocol we developed to preserve the phosphorylation status of COX, we show that heart Cyt $c$ is phosphorylated in vivo without further experimental manipulation of a cellular signaling pathway.

Western blots with anti-phospho antibodies indicated tyrosine phosphorylation of our purified Cyt $c$ (Figure 1B, lane 4). We also obtained a weak signal (not shown) with two sets of anti-phosphoserine and -threonine antibodies (see Materials and Methods). However, in contrast to the highly specific 4G10 anti-phosphotyrosine antibody, anti-phosphoserine and -threonine antibodies are known to be less specific and may also cross-react with phosphorylated tyrosine residues. Applying mass spectrometry, we unambiguously identified the only phosphorylated residue as Tyr-97 (Figure 2).

We have preliminarily assessed one functional consequence of Cyt $c$ phosphorylation by analyzing COX activity. Phosphorylated Cyt $c$ produces enhanced sigmoidal COX kinetics, whereas a hyperbolic response is obtained using SAP-treated Cyt $c$ (Figure 4). Interestingly, the maximal turnover is identical with phosphorylated and SAP-treated Cyt $c$, possibly due to the presence of unphosphorylated Cyt $c$ in our purification. Even though our isolation approach produced a single Cyt $c$ fraction, we were also able to detect unphosphorylated Tyr-97 by mass spectrometry (not shown). When high-resolution SDS-PAGE was applied, only a single band was obtained for phosphorylated and SAP-treated Cyt $c$ samples, each running at the same position (not shown), and it remained elusive what part of our isolated Cyt $c$ was phosphorylated. However, for the reaction with COX, the $K_{\mathrm{m}}$ for phosphorylated Cyt $c$ was about 2-fold higher compared to dephosphorylated Cyt $c$, and about 2.2 times more in vivo phosphorylated Cyt $c$ was required to reach maximal turnover compared to dephosphorylated Cyt $c$ (Figure 4). The clear differences in enzyme kinetics together with the spectral changes (Figure 3 ) for phosphorylated Cyt $c$ suggest that a substantial fraction of the isolated Cyt $c$ carries a phosphate group. A major future goal will be the separation of Tyr-97-phosphorylated and -unphosphorylated 


$\begin{array}{crcr}\text { Cyt } & c & 93 & \text { DLIAYLKKAT } \\ & : \quad:=\quad:: \\ \text { COX I } & 300 & \text { DTRAYFTSAT }\end{array}$

FIGURE 5: Sequence alignment of the phospho-epitopes of Cyt $c$ and COX subunit I. Five out of 10 residues are identical in both epitopes. In contrast to the COX sequence, which is invariant in all eukaryotes (14), the sequence in Cyt $c$ shows some variation between species, including the absence of Tyr-97 in the mold Neurospora crassa (accession number X05506).

Cyt $c$ species. Once this has been achieved, additional functional aspects of Cyt $c$ phosphorylation can be evaluated, including the effect on redox potential, reaction with the $b c_{1}$ complex, and the possible role in apoptosis.

Tyr-97 is conserved in most species ranging from mammals to microorganisms and plants, with Neurospora crassa as an exception containing a Phe residue instead $(3,32)$. There are also two known yeast mutants where the corre- sponding Tyr was changed into Leu and Gln, which retained Cyt $c$ expression and function (33). The finding that those changes do not appear to be crucial for organism survival may be readily explained with the lack of tyrosine kinase signaling in these organisms, because no tyrosine kinases have been identified in yeast and $N$. crassa.

Comparison of the sequence surrounding the phosphorylation site in COX subunit I (14) with the epitope of heart Cyt $c$ presented here revealed that 5 of 10 residues are identical (Figure 5). The alanine residue immediately adjacent to the phosphorylated tyrosines in $\mathrm{COX}$ and $\mathrm{Cyt} c$ contrasts with acidic residues commonly found in tyrosine kinase recognition motifs in this position (34). Furthermore, the overlay of the two domains based on the crystal structures produced a good fit for the backbone of all amino acids and also for the side chains of the five identical residues (Figure $6 \mathrm{~B})$. The motif comprised of those five residues only occurs
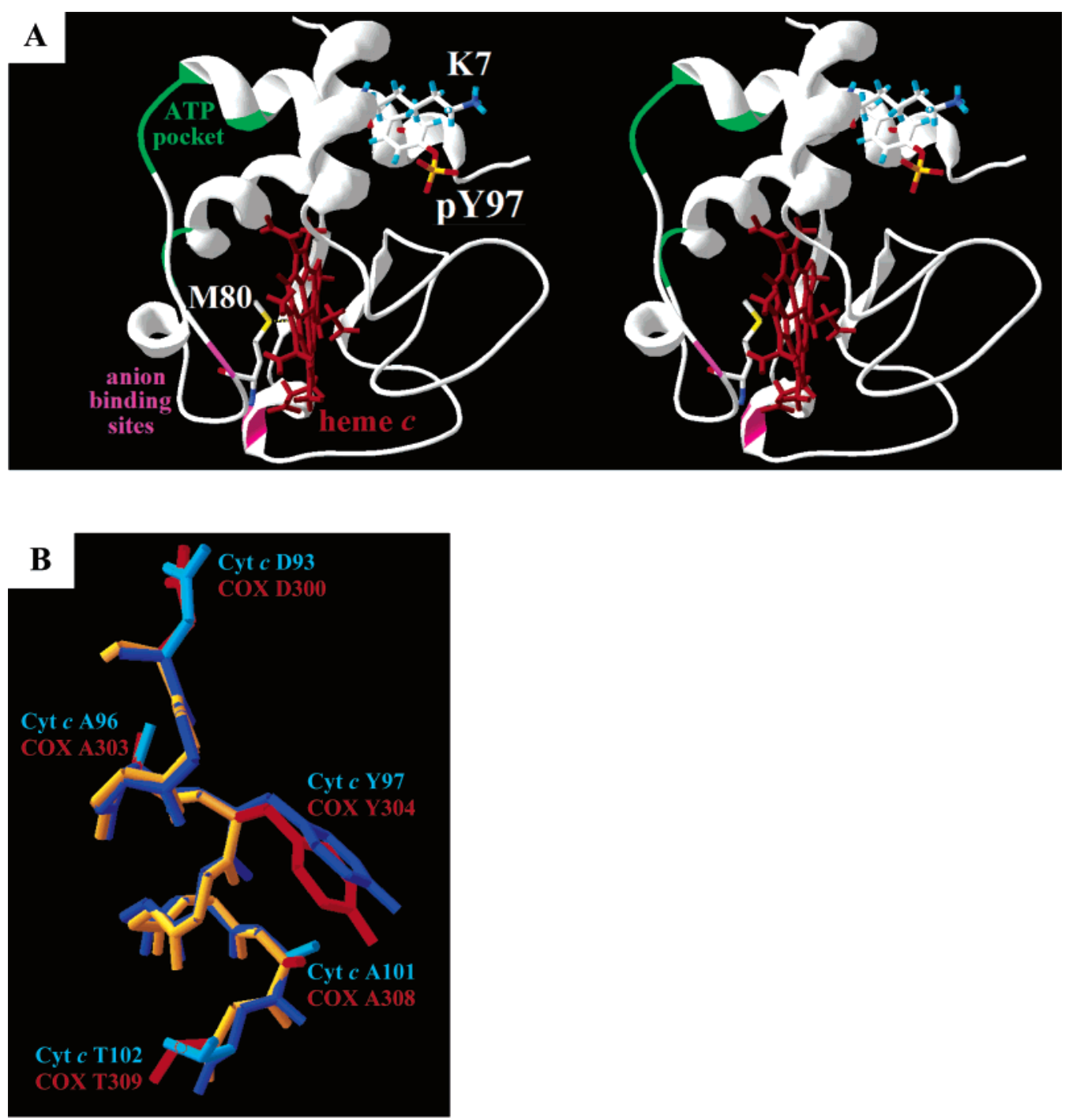

FIGURE 6: Conventional view of the cytochrome $c$ crystal structure. Crystallographic data from horse Cyt $c$ (23), which has the same amino acid sequence as cow, was used. A phosphate group was attached to Tyr-97 using the program Hyperchem. A geometrically optimized spatial arrangement for the modeled phosphotyrosine side chain was obtained applying 500 cycles of the 'steepest descent' algorithm. Modeled Cyt $c$ was processed with the program Swiss PDB viewer (version 3.7). (A) Stereo view of the modeled Cyt $c$. Phosphotyrosine 97 is located on the "right side" of the molecule (highlighted in sticks). The distance between the modeled phosphate group and the $\epsilon$ - $\mathrm{NH}_{3}$ group of Lys-7 (sticks), a key residue for apoptosome formation, is $>5 \AA$. Residues Glu-69, Asn-70, Lys-72, Lys-86, Lys-87, and Lys-88 that form an ATP binding pocket (12) are indicated in green ribbon. Residues Asn-51 and Ile-81 involved in anion binding (3) are indicated in magenta ribbon. The bond between Met-80 (sticks) and the heme iron produces the characteristic $695 \mathrm{~nm}$ absorption band. (B) Overlay of the phospho-epitopes of Cyt $c$ and $\operatorname{COX}(14,24)$ was performed with the iterative magic fit function. Identical amino acids are highlighted in sticks. 
in COX subunit I and Cyt $c$ among ETC complexes, and it is possible that both proteins are targeted by the same kinase and/or phosphatase. Since Cyt $c$ and COX directly interact with each other, it is noteworthy that Tyr-97 is not directly involved in Cyt $c$-COX binding (35).

Ferricytochrome $c$ binds anions, including phosphate that also binds to the ferro form and shows the highest binding affinity, followed by chloride, iodide, sulfate, and cacodylate (36). On the basis of crystal structures, the generally hydrophobic environment of Tyr-97 located on the conventional "right side" of the molecule does not contain a phosphate, but two anion binding sites have been identified on the "left side" of the molecule in the region of Ile-81 and Asn-51 (3) (Figure 6A). Although localized to the same side of the molecule as Met-80, binding of those anions does not cause spectral changes (37), including the characteristic 695 $\mathrm{nm}$ band. Met- 80 is a heme iron ligand, and this bond causes a $695 \mathrm{~nm}$ absorption band. Only functional Cyt $c$ shows this characteristic band, which is a marker for Cyt $c$ integrity, and changes in the $695 \mathrm{~nm}$ band may indicate different functional states of Cyt $c$ (26). As we have shown, Cyt $c$ purified under conditions that maintain the physiological phosphorylation status shows a shift of this band to $687 \mathrm{~nm}$ (Figure 3), indicating subtle structural changes in the heme environment. This was unexpected because Met-80 and Tyr97 are located on opposite hemispheres of the molecule (Figure 6A). That the spectral change is a result of phosphorylation rather than any other possible post-translational modification is further supported by our finding that removal of the phosphate groups via shrimp alkaline phosphatase treatment (Figure 1B, lane 5) produces a spectrum similar to commercially available Cyt $c$ (Figure 3).

Tyr-97 is located in the middle of a relatively hydrophobic area of the otherwise highly charged Cyt $c$, a region that has been suggested to serve as a binding site for hydrophobic moieties (3), such as a lipid. A large body of literature exists on the interaction of Cyt $c$ with acidic phospholipids such as those that are present in the mitochondrial inner membrane, and there are two distinct binding sites (38 and references therein): an anion sensitive A-site likely involving the ATP binding site located on the "left side" on Cyt $c$ (Figure 6A), and the hydrophobic $\mathrm{C}$-site (C for cardiolipin), which provides strong membrane association of Cyt $c$ at 100 $\mathrm{mM} \mathrm{NaCl}$ via binding to the mitochondrial phospholipid cardiolipin (38). Notably, a recent study has revealed an additional function of Cyt $c$ as a cardiolipin oxygenase (10): after induction of apoptosis, Cyt $c$ chemically oxidizes cardiolipin, allowing its detachment and release from the mitochondria. Such oxidation through Cyt $c$ involves a tyrosine radical (10). This novel function requires lipid binding capability and a tyrosine residue, two features provided by the Tyr-97 region. In addition, Tyr-97 is connected to the heme moiety through a channel that shows a conduit of hydrophobicity (3), possibly capable of electron transfer as required for the cardiolipin redox chemistry.

It is interesting to speculate about further consequences of Cyt $c$ phosphorylation: since we have found that Cyt $c$ is tyrosine-phosphorylated in vivo, this might represent the functional state in healthy heart tissue. Such phosphorylation could modulate binding of cardiolipin to the hypothetical binding site on Cyt $c$ or prevent cardiolipin oxidation if Tyr97 is involved in this process. Interestingly, the side chain of Cyt $c$ Lys-7, a key residue that participates in apoptosome formation (39), is spatially located next to the side chain of Tyr-97 (Figure 6A). Both residues may form a salt bridge, which could hinder apoptosome formation. Given the homology between the phosphorylation epitopes in Cyt $c$ and COX, and our finding that $\mathrm{COX}$ is targeted for phosphorylation by the cAMP-dependent pathway in liver, it is possible that the same pathway targets Cyt $c$. If so, this may in part explain why the cAMP pathway is anti-apoptotic in most tissues (40). It would perhaps also change the dominant view that the role of Cyt $c$ in apoptosis is as a passive byproduct of mitochondrial damage instead of as an active participant regulated by signaling pathways.

\section{ACKNOWLEDGMENT}

We thank Drs. Bernhard Kadenbach and Shelagh Ferguson-Miller for comments on the manuscript.

\section{REFERENCES}

1. MacMunn, C. (1886) Researches on myohaematin and the histohaematins, Philos. Trans. R. Soc. London 177, 267-298.

2. Keilin, D. (1925) On cytochrome, a respiratory pigment, common to animals, yeast, and higher plants, Proc. R. Soc. London, Ser. B 98, 312-339

3. Dickerson, R. E., Takano, T., Eisenberg, D., Kallai, O. B., Samson, L., Cooper, A., and Margoliash, E. (1971) Ferricytochrome $c$. I. General features of the horse and bonito proteins at $2.8 \AA$ resolution, J. Biol. Chem. 246, 1511-1535.

4. Li, K., Li, Y., Shelton, J. M., Richardson, J. A., Spencer, E., Chen, Z. J., Wang, X., and Williams, R. S. (2000) Cytochrome $c$ deficiency causes embryonic lethality and attenuates stress-induced apoptosis, Cell 101, 389-399.

5. Morriss, G. M., and New, D. A. (1979) Effect of oxygen concentration on morphogenesis of cranial neural folds and neural crest in cultured rat embryos, J. Embryol. Exp. Morphol. 54, 1735 .

6. Green, D. R. (2000) Apoptotic pathways: paper wraps stone blunts scissors, Cell 102, 1-4.

7. Bernardi, P., Petronilli, V., Di Lisa, F., and Forte, M. (2001) A mitochondrial perspective on cell death, Trends Biochem. Sci. 26, $112-117$.

8. Kroemer, G., Dallaporta, B., and Resche-Rigon, M. (1998) The mitochondrial death/life regulator in apoptosis and necrosis, Annu. Rev. Physiol. 60, 619-642.

9. Skulachev, V. P. (1998) Cytochrome $c$ in the apoptotic and antioxidant cascades, FEBS Lett. 423, 275-280.

10. Kagan, V. E., Tyurin, V. A., Jiang, J., Tyurina, Y. Y., Ritow, V. B., Amoscato, A. A., Osipov, A. N., Belikova, N. A., Kapralov, A. A., Kini, V., Vlasova, I. I., Zhao, Q., Zou, M., Di, P., Svistunenko, D. A., Kurnikov, I. V., and Borisenko, G. G. (2005) Cytochrome $c$ acts as a cardiolipin oxygenase required for release of proapoptotic factors, Nat. Chem. Biol. 1, 223-232.

11. Pereverzev, M. O., Vygodina, T. V., Konstantinov, A. A., and Skulachev, V. P. (2003) Cytochrome $c$, an ideal antioxidant, Biochem. Soc. Trans. 31, 1312-1315.

12. McIntosh, D. B., Parrish, J. C., and Wallace, C. J. (1996) Definition of a nucleotide binding site on cytochrome $c$ by photoaffinity labeling, J. Biol. Chem. 271, 18379-18386.

13. Ferguson-Miller, S., Brautigan, D. L., and Margoliash, E. (1976) Correlation of the kinetics of electron-transfer activity of various eukaryotic cytochromes $\mathrm{c}$ with binding to mitochondrial cytochrome $c$ oxidase, J. Biol. Chem. 251, 1104-1115.

14. Lee, I., Salomon, A. R., Ficarro, S., Mathes, I., Lottspeich, F., Grossman, L. I., and Hüttemann, M. (2005) cAMP-dependent tyrosine phosphorylation of subunit I inhibits cytochrome $c$ oxidase activity, J. Biol. Chem. 280, 6094-6100.

15. Miyazaki, T., Neff, L., Tanaka, S., Horne, W. C., and Baron, R. (2003) Regulation of cytochrome $c$ oxidase activity by c-Src in osteoclasts, J. Cell Biol. 160, 709-718.

16. Schilling, B., Aggeler, R., Schulenberg, B., Murray, J., Row, R. H., Capaldi, R. A., and Gibson, B. W. (2005) Mass spectrometric identification of a novel phosphorylation site in subunit NDUFA10 of bovine mitochondrial complex I, FEBS Lett. 579, 2485-2490. 
17. Zhang, F. X., Pan, W., and Hutchins, J. B. (1995) Phosphorylation of $\mathrm{F}_{1} \mathrm{~F}_{0}$ ATPase delta-subunit is regulated by platelet-derived growth factor in mouse cortical neurons in vitro, J. Neurochem. $65,2812-2815$.

18. Ko, Y. H., Pan, W., Inoue, C., and Pedersen, P. L. (2002) Signal transduction to mitochondrial ATP synthase: evidence that PDGFdependent phosphorylation of the delta-subunit occurs in several cell lines, involves tyrosine, and is modulated by lysophosphatidic acid, Mitochondrion 1, 339-348.

19. Kadenbach, B., and Urban, P. F. (1968) Application of a quantitative chromatographic method of purification in the study of the biosynthesis of cytochrome c., Z. Anal. Chem. 243, 542554.

20. Ficarro, S. B., McCleland, M. L., Stukenberg, P. T., Burke, D. J., Ross, M. M., Shabanowitz, J., Hunt, D. F., and White, F. M. (2002) Phosphoproteome analysis by mass spectrometry and its application to Saccharomyces cerevisiae, Nat. Biotechnol. 20, 301-305.

21. Ficarro, S. B., Salomon, A. R., Brill, L. M., Mason, D. E., StettlerGill, M., Brock, A., and Peters, E. C. (2005) Automated immobilized metal affinity chromatography/nano-liquid chromatography/electrospray ionization mass spectrometry platform for profiling protein phosphorylation sites, Rapid Commun. Mass Spectrom. 19, 57-71.

22. Eng, J., McCormack, A., and Yates, J. R. (1994) An approach to correlate tandem mass spectral data of peptides with amino acid sequences in a protein database, J. Am. Soc. Mass Spectrom. 5, 976-989.

23. Sanishvili, R., Volz, K. W., Westbrook, E. M., and Margoliash, E. (1995) The low ionic strength crystal structure of horse cytochrome $c$ at $2.1 \AA$ resolution and comparison with its high ionic strength counterpart, Structure 3, 707-716.

24. Tsukihara, T., Shimokata, K., Katayama, Y., Shimada, H., Muramoto, K., Aoyama, H., Mochizuki, M., Shinzawa-Itoh, K., Yamashita, E., Yao, M., Ishimura, Y., and Yoshikawa, S. (2003) The low-spin heme of cytochrome $c$ oxidase as the driving element of the proton-pumping process, Proc. Natl. Acad. Sci. U.S.A. 100, 15304-15309.

25. Bushnell, G. W., Louie, G. V., and Brayer, G. D. (1990) Highresolution three-dimensional structure of horse heart cytochrome c, J. Mol. Biol. 214, 585-595.

26. Dickerson, R., and Timkovich, R. (1975) in The Enzymes (Boyer, P., Ed.) pp 397-472, Academic Press, New York.

27. Ando, K., Matsubara, H., and Okunuki, K. (1966) Alkylation of cytochromes c. II. Carboxymethylation of beef and human cytochromes $c$ in the oxidized and reduced forms, Biochim. Biophys. Acta 118, 256-267.
28. Stellwagen, E. (1968) Carboxymethylation of horse heart ferricytochrome $c$ and cyanferricytochrome $c$, Biochemistry 7, 24962501.

29. Schejter, A., and Aviram, I. (1970) The effects of alkylation of methionyl residues on the properties of horse cytochrome $c, J$. Biol. Chem. 245, 1552-1557.

30. Brunori, M., Wilson, M. T., and Antonini, E. (1972) Properties of modified cytochromes. I. Equilibrium and kinetics of the $\mathrm{pH}$ dependent transition in carboxymethylated horse heart cytochrome c, J. Biol. Chem. 247, 6076-6081.

31. Keller, R. M., Aviram, I., Schejter, A., and Wuthrich, K. (1972) Evidence for pentacoordinated iron (II) in carboxymethylated cytochrome $c$, FEBS Lett. 20, 90-92.

32. Heller, J., and Smith, E. L. (1966) Neurospora crassa cytochrome c. II. Chymotryptic peptides, tryptic peptides, cyanogen bromide peptides, and the complete amino acid sequence, J. Biol. Chem. 241, 3165-3180.

33. Hampsey, D. M., Das, G., and Sherman, F. (1988) Yeast iso-1cytochrome $c$ : genetic analysis of structural requirements, FEBS Lett. 231, 275-283.

34. Pearson, R. B., and Kemp, B. E. (1991) Protein kinase phosphorylation site sequences and consensus specificity motifs: tabulations, Methods Enzymol. 200, 62-81.

35. Roberts, V. A., and Pique, M. E. (1999) Definition of the interaction domain for cytochrome $c$ on cytochrome $c$ oxidase. III. Prediction of the docked complex by a complete, systematic search, J. Biol. Chem. 274, 38051-38060.

36. Barlow, G. H., and Margoliash, E. (1966) Electrophoretic behavior of mammalian-type cytochromes c, J. Biol. Chem. 241, 14731477.

37. Nicholls, P. (1974) Cytochrome $c$ binding to enzymes and membranes, Biochim. Biophys. Acta 346, 261-310.

38. Rytomaa, M., and Kinnunen, P. K. (1994) Evidence for two distinct acidic phospholipid-binding sites in cytochrome $c, J$. Biol. Chem. 269, 1770-1774

39. Yu, T., Wang, X., Purring-Koch, C., Wei, Y., and McLendon, G. L. (2001) A mutational epitope for cytochrome $c$ binding to the apoptosis protease activation factor-1, J. Biol. Chem. 276, 1303413038.

40. Martin, M. C., Allan, L. A., Lickrish, M., Sampson, C., Morrice, N., and Clarke, P. R. (2005) Protein kinase A regulates caspase-9 activation by Apaf-1 downstream of cytochrome $c, J$. Biol. Chem. $280,15449-15455$.

BI060585V 